\title{
A comparison of two diffusion process models in accounting for payoff and stimulus frequency manipulations
}

\author{
Fábio P. Leite
}

Published online: 6 June 2012

(C) Psychonomic Society, Inc. 2012

\begin{abstract}
I analyzed response time and accuracy data from a numerosity discrimination experiment in which both stimulus frequency and payoff structure were manipulated. The numerosity discrimination encompassed responding either "low" or "high" to the number of asterisks in a $10 \times 10$ grid, on the basis of an experimenter-determined decision cutoff (fixed at 50). In the stimulus frequency condition, there were more low than high stimuli in some blocks and more high than low stimuli in other blocks. In the payoff condition, responses were rewarded such that the relative value of a stimulus mimicked the relative frequency of that stimulus in the previous manipulation. I modeled the data using two sequential-sampling models in which evidence was accumulated until either a "low" or a "high" decision criterion was reached and a response was initiated: a single-stage diffusion model framework and a two-stage diffusion model framework. In using these two frameworks, the goal was to examine their relative merits across stimulus frequency and payoff structure manipulations. I found that shifts in starting point in a single-stage diffusion framework and shifts in the initial drift rate in the two-stage model were able to account for the data. I also found, however, that these two shifts across the two models produced similar changes in the random walk that described the decision process. In conclusion, I found that the similarities in the descriptions of the decision processes make it difficult to choose between the two models and suggested that such a choice should consider model assumptions and parameter estimate interpretations.
\end{abstract}

F. P. Leite $(\bowtie)$

Department of Psychology, Ohio State University,

Lima, OH 45804, USA

e-mail: leite.11@osu.edu
Keywords Response time - Diffusion model · Two-stage model $\cdot$ Payoff $\cdot$ Stimulus frequency

Studying the effects of payoff manipulations has been of longstanding interest in cognitive psychology (e.g., Edwards, 1965; Fitts, 1966). In manipulations involving payoffs mapped to two choices, for example, participants can be induced to favor the correct response alternative over the incorrect response because of a high reward (or a low cost) value associated with the correct response, or because of a high cost (or a low reward) value associated with the incorrect response. This type of manipulation is often implemented with a performancedriven, variable monetary reward to the participant (see Chiew \& Braver, 2011; Dambacher, Hübner, \& Schlösser, 2011, for recent studies of the factors that determine under what conditions monetary incentives enhance performance).

Consistent with the description above, Diederich and Busemeyer (2006) used a line length judgment to examine how sequential-sampling models might represent the effects of payoffs on mean response time (RT). They compared three hypotheses: bound change, in which only the starting position of the accumulation process is influenced by the payoffs, but the accumulation of information extracted from the stimuli is not (cf. Link \& Heath, 1975); drift rate change, in which the payoffs influence the rate of information accumulation (cf. Ashby, 1983; Ratcliff, 1981); and two-stage processing, in which the decision process consists of two subprocesses, switching from one that processes payoffs to another that processes the stimulus (cf. Heath, 1981, 1992; Laming, 1968; Ratcliff, 1980). Diederich and Busemeyer found that two-stage processing provided a better account of the data than did either drift-rate change or bound change, suggesting that individuals initially process the payoff 
information, then switch to the stimulus information (p. 206; see also Busemeyer \& Diederich, 2002; Diederich, 1995, 1997, 2008).

Like manipulations of payoffs, manipulations of stimulus frequency cause participants to alter their responses, now on the basis of the probability of occurrence of the stimulus, such that they are faster and more accurate when responding to stimuli that appear more often than to those that appear less often (Falmagne, 1965; Jarvik, 1951; Kirby, 1976; Laming, 1969; Remington, 1969).

Examining these two manipulations against one another, Leite and Ratcliff (2011) reported data involving a numerosity discrimination in which participants judged the number of asterisks in a grid as either high or low (according to an experimenter-defined criterion) and in which the stimulus relative frequency and the response payoffs were manipulated.

Leite and Ratcliff's (2011) examination in the context of perceptual decision making with two alternatives followed that of Green and Swets (1966) in the context of signal detection theory (see also Swets, Tanner, \& Birdsall, 1961). When describing signal detection theory in terms of a choice between two alternatives, it is assumed that the observer sets a threshold criterion that the relative amplitude of one alternative's signal must surpass so that a response is made in favor of that alternative. It is further assumed that the model parameter associated with the threshold criterion measures the response bias for which it accounts and that this criterion setting is influenced by the prior probability of the signal and payoffs for each type of error (viz., responding Alternative 1 when Alternative 2 is present, or vice versa). Because signal detection theory cannot account for accuracy and RT at the same time (i.e., it gives a fixed-sample description of the decision), researchers have turned to sequentialsampling models, among other families of models, to fit both dependent variables simultaneously. The assumptions used by sequential-sampling models, however, are equivalent to the assumptions described above in the signal detection model.

In the present article, I used the data from Leite and Ratcliff's (2011) Experiment 2 to examine the relative merits of the two-stage model (Diederich \& Busemeyer, 2006) and the (Wiener) diffusion model (Ratcliff, 1978; Ratcliff \& Rouder, 1998) across stimulus frequency and payoff structure manipulations. The present model comparison extended that of Diederich and Busemeyer (2006) in two important ways: It examined how the models might represent the effects of payoffs on the entire RT distributions of both correct and error responses, vis-à-vis their representations of stimulus frequency effects (see Ratcliff \& Murdock, 1976, for a discussion of the necessity of examining RT distributions when evaluating RT models), and it examined how the models might represent the effects of payoffs and stimulus frequency in a different task.

\section{Computational models}

Several families of computational models have aided our understanding of perceptual decision making by providing a theoretical background against which to infer the properties of the underlying sensory representation and the processing leading to decisions. To account simultaneously for accuracy and RT, researchers have turned to, among other families of models, sequential-sampling models-following Stone's (1960) original random-walk model proposal (later extended by Laming, 1968, such that error responses were not bound to be faster than correct responses) and LaBerge's (1962) recruitment model (later extended by Vickers, 1970, such that observed RTs were not systematically overestimated).

One well-known sequential-sampling model is the diffusion process model (Ratcliff, 1978). In the diffusion model, noisy evidence is accumulated over time toward one of two response criteria that correspond to the two possible responses. This accumulation starts at a point subject to across-trial variability, and when a criterion is reached, the accumulation process is terminated and a response is executed (see Fig. 1, top panel, for an illustration). Diederich and Busemeyer's (2006) two-stage model is another sequential-sampling model, applied to experiments with different payoffs between alternatives. Its architecture (see Fig. 1, bottom panel) is similar to the diffusion model's, but it includes two accumulation processes toward one of the two response criteria: one that operates, always first, during the processing of an attribute (e.g., the payoff scheme), always starting at the midpoint between the two criteria, and a

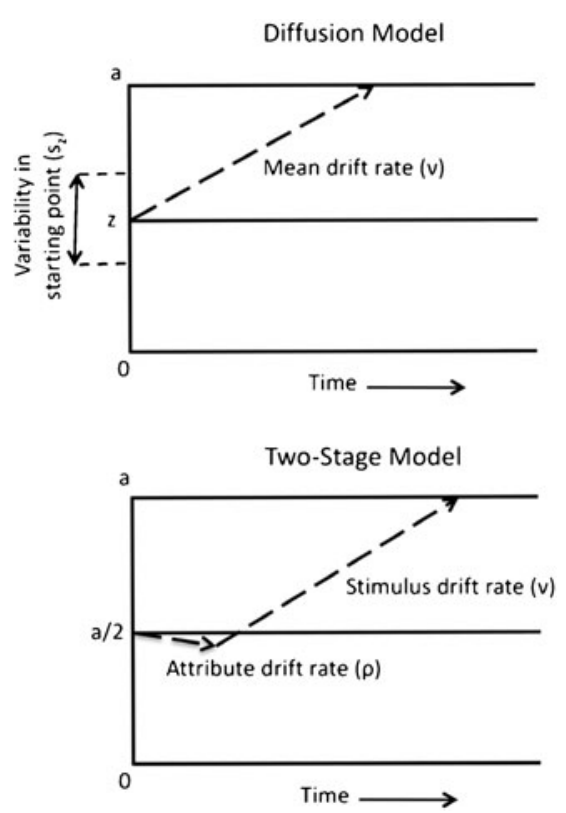

Fig. 1 Illustration of the models. The top panel shows a sketch of the diffusion model in which $\nu$ (dashed line) represents the mean drift rate associated with a particular stimulus for which accumulation starts at $z$. The boundaries (or decision thresholds) are represented by 0 and $a$. The bottom panel shows an analogous sketch of the two-stage model 
second that operates later, during the processing of stimulus evidence.

In both models, stimulus evidence is assumed to accumulate gradually over time, from a starting point toward one of two alternative decision boundaries. The separation between these two boundaries, or the amount of evidence required for a response, is modeled by parameter $a$ (equivalent to one of the boundaries, because the other is set at 0 ). The easier the discrimination between the two alternatives, the faster the accumulation process reaches a boundary, at which point the accumulation is terminated and a response is made.

In the diffusion model, the rate at which information is accumulated is a function of the ease of discrimination (i.e., the relative quality of stimulus information) - represented by, the drift rate $\nu$; in the two-stage model, that rate is a function of both the attribute information (viz., stimulus frequency or payoffs) - the drift rate $\rho$-and the relative quality of stimulus information-the drift rate $\nu$.

In both models, variability in the accumulation of information within a trial (i.e., noise) gives rise to variability in RTs and allows the process to hit the wrong boundary, giving rise to errors. In the diffusion model, across-trial variability in the values of stimulus-driven drift rates $(\eta)$ and starting point $\left(s_{z}\right)$ is used, because it is assumed that participants do not set identical starting points and that the same stimulus does not map onto identical drift rate values from trial to trial (e.g., Laming, 1968; Ratcliff, 1978). Table 1 summarizes the function and the label of each parameter in both models.

Although the models are similar in architecture, one significant theoretical difference between the two-stage model and the diffusion model involves the assumptions about when information relevant to the decision between the two alternatives (but not presented with the stimulus, such as payoff information) is processed. On the one hand, the diffusion model's framework assumes that information such as payoff is processed prior to the processing of information from the stimulus. That is, knowledge of the payoff associated with responses will influence how participants set the starting point of the evidence accumulation or the threshold that the accumulation process must reach before a decision is made. On the other hand, the two-stage model's framework assumes that payoff information is processed during the accumulation of evidence toward a decision, immediately preceding the processing of information extracted directly from the stimulus (which typically corresponds to a higher drift rate than the one associated with the stimulus attribute).

In the two-stage model, the amount of time during which the accumulation process will operate in the first stage is determined by a switching-probability parameter. That is, in each iteration of the random walk (driven by an attributebased drift rate), the first stage has a small probability of terminating (and being replaced by the stimulus-based accumulation process). This leads to two possible scenarios: one, a simulated trial in the model will terminate without transitioning to the second stage; and, two, the attribute-based drift rate will be replaced by the stimulus-based drift rate, with the resulting predicted RTs being influenced by the drift rates in both stages. Although the first scenario is the exception, it is of importance because it means that when it happens, the twostage model is generating predictions in the same manner as the diffusion model (with the starting point set to the midpoint between the two criteria).

Leite and Ratcliff (2011) showed that response bias due to changes in stimulus frequency was accounted for in the diffusion model by moving the starting point of the accumulation process closer to the boundary associated with the most frequent alternative; response bias due to changes in payoffs was accounted for by a similar but smaller shift in starting point. In the two-stage model, response bias due to changes in payoffs can be accounted for by nonzero drift parameters governing

Table 1 Model parameters

\begin{tabular}{|c|c|c|c|}
\hline \multirow[b]{2}{*}{ Parameter } & $\mathrm{DM}$ & $2-\mathrm{S}$ & \multirow[b]{2}{*}{ Short Description } \\
\hline & \multicolumn{2}{|c|}{ Represented as } & \\
\hline Boundary separation & $a$ & $a$ & Distance between the boundaries corresponding to the two alternatives (viz., high and low). \\
\hline Nondecision time & $T_{\mathrm{er}}$ & $T_{\mathrm{er}}$ & Time (in seconds) devoted to processes such as encoding and initiating motor response. \\
\hline Variability in $T_{\mathrm{er}}$ & $s_{t}$ & & Range associated with cross-trial variability in $T_{\mathrm{er}}$. \\
\hline Stimulus drift rate & $\nu_{1-6}$ & $\nu_{1-6}$ & Mean drift rates associated with the stimuli in each of the six levels of difficulty. \\
\hline Variability in $\nu$ & $\eta$ & & Cross-trial variability in perceptual strength (to similar stimuli). \\
\hline Starting point & $z_{1}, z_{\mathrm{h}}$ & $a / 2$ & Where accumulation of evidence toward one of the boundaries starts. \\
\hline Variability in $z$ & $s_{z}$ & & Range associated with cross-trial variability in $z$. \\
\hline Attribute drift rate & & $\rho_{\mathrm{l}}, \rho_{\mathrm{h}}$ & Mean drift rates associated with the payoff or frequency information. \\
\hline Switching probability & & swt & Probability that the driving parameter of the accumulation process switches from $\rho$ to $\nu$. \\
\hline
\end{tabular}

For each of the last four parameters described, there were different free parameters across Conditions 1 and 2. DM refers to the diffusion model; 2-S refers to the two-stage model 
the processing of the first attribute, provided that no boundary is reached during that stage; response bias due to changes in stimulus frequency can be accounted for by a similar process. ${ }^{1}$

In the present article, I will examine the relative differences in fitting data subject to stimulus frequency and payoff structure manipulations by assuming that these manipulations affect starting point (in the diffusion model) or an initial stage of accumulation of evidence that is governed by a stimulus attribute (i.e., the two-stage model's first stage). This involves comparing model predictions based on a single-stage diffusion process with model predictions based on a two-stage process in which one or two drift rates drive the accumulation process - in effect, creating a convolution between single-stage processes starting at the midway point between two boundaries and a two-stage process in which the stimulus-driven accumulation potentially starts closer to one of the two boundaries after an initial delay (corresponding to the length of the first stage).

By focusing on these two models, I am assuming that sequential-sampling models are reasonable approximations of the cognitive processes driving two-choice decisions. Despite following several reports that share this assumption (Ashby, 1983; Diederich, 2008; Diederich \& Busemeyer, 2006; Leite \& Ratcliff, 2011; Ratcliff \& Rouder, 1998; Ratcliff \& Smith, 2004), however, the assumption could be challenged. Laming (1968), for example, proposed that participants may initiate the decision process before any stimulus information is available: Because there is no stimulus, the random walk starts with zero drift, and then the stimulus information changes the drift rate of the process. (Such "false starts" could distance any model from the true state of the decision process.) Furthermore, Laming (2010, sec. 5) also challenged the notion that two-choice RTs are information-limited. Specifically, he showed that a simple random-walk model for choice RT does not adequately account for pragmatic trial-to-trial adjustments based upon the stimulus presented and the response made on each trial, which may drive participants' adjustments to stimulus frequency or payoff structure (see also Laming, 1968, chap. 8).

\section{Method}

Overview of experiment 2 in Leite and Ratcliff (2011)

The experimental task was a two-choice numerosity discrimination in which participants were asked to respond, as quickly and as accurately as possible, either "low" or

\footnotetext{
${ }^{1}$ Because the two-stage model assumes processing of some attribute (i.e., not the stimulus) in the first stage, the information about the unequal stimulus frequency was provided to the participants (at the beginning of each block of trials).
}

"high" to the number of asterisks in a $10 \times 10$ grid, relative to 50 asterisks. The task structure was manipulated across blocks of trials by changing either the frequency (Condition 1) or the payoff scheme (Condition 2) associated with each type of response (akin to manipulations described in Green \& Swets, 1966 , sec. 4.2 .1 , to induce the participant to change the decision criterion). Such a numerosity discrimination is a simple task for participants to perform, and it provides the experimenter with the ability to manipulate stimulus frequency and to incorporate payoffs easily. In addition, there are few perceptual or memory limitations to the task, to which both sequential-sampling models were expected to be successfully applied (based on results in, for example, Ratcliff, Thapar, \& McKoon, 2001).

In Condition 1, the proportion of low to high stimuli was manipulated, such that there were blocks of trials with three times as many low as high stimuli, and vice versa. Before beginning each block, the participants were informed of how many low and high stimuli would be present (e.g., "The following block contains 27 low stimuli and nine high stimuli."). This setup allowed participants to adjust to the relative frequencies prior to processing the stimulus information, which matches naturally to adjustments in starting point in the diffusion model. In the two-stage model, the assumption was that the initial drift would drive the accumulation while participants were thinking of the relative frequencies as the stimulus came on.

In Condition 2, the changes in payoff structure mimicked the changes in stimulus frequency, with participants being instructed to maximize their score. Prior to beginning each block, the participants were informed of the payoff structure (e.g., "In the following block, 27 points will be added to your score for each correct 'low' response, and nine points will be deducted from your score for each incorrect 'low' response. Conversely, nine points will be added to your score for each correct 'high' response, and 27 points will be deducted from your score for each incorrect 'high' response."). In the low-biased blocks, the participants received three times as many points for answering "low" correctly as were deducted for answering "low" incorrectly; likewise, they had three times as many points deducted for answering "high" incorrectly as were received for answering "high" correctly. In the high-biased blocks, the reverse point structure applied. After each trial, the number of points received or deducted was shown to the participants; a running score (in points) was shown after each block. In each block, half of the trials consisted of low stimuli and half consisted of high stimuli. The feedback on how many points were added to or subtracted from the running score shown after each trial allowed the participants to think of the point structure 
shown in the beginning of the block, in line with Diederich and Busemeyer's (2006) design (in which the payoff structure was repeated before each trial).

Six Ohio State University students completed five experimental sessions (each lasting approximately $40-45 \mathrm{~min}$ ) in a $4 \times 3 \times 2 \times 2$ within-subjects design - with Task, Difficulty, Bias Level, and Type of Response (low or high) as factors, respectively. Participants were compensated at the rate of $\$ 8.00$ per session, plus bonus compensation contingent upon performance in the payoff structure condition (for performance between Sessions 2 and 5, which averaged $\$ 1.09$ per session). I chose to model the data from Experiment 2 only because it minimized the individual differences in performance and the model parameter estimates observed in Experiment 1.

\section{Modeling analysis}

The diffusion model and the two-stage model were fitted to each individual participant's data, modeling the two bias levels and both error and correct RT distributions simultaneously. The RT data were divided into three levels of difficulty, as determined by the number of asterisks present in each stimulus: $36-40$ (vs. 61-65), 41-45 (vs. 56-60), and 46-50 (vs. 51-55). The RT distributions were approximated by five quantiles, evenly spaced between .1 and .9 .

I fitted the models to the accuracy and RT data-divided into six bins defined by the five evenly spaced quantiles above, for correct and error responses - using a minimization routine based on the simplex fitting method (Nelder \& Mead, 1965). The quantile RTs and the diffusion model were used to generate the predicted cumulative probability of a response by that quantile RT. Subtracting the cumulative probabilities for each successive quantile from that of the next higher quantile gives the proportion of responses between adjacent quantiles. For the chi-squared $\left(\chi^{2}\right)$ computation, these are the expected values to be compared to the observed proportions of responses between the quantiles (i.e., .1, .2, .2, .2, .2, and .1), multiplied by the number of observations in each condition. Summing over (observed expected $)^{2}$ /expected for all conditions gives the chi-square value to be minimized (Eq. 1; cf. Leite \& Ratcliff, 2011):

$\chi^{2}=\sum_{k=1}^{2} \sum_{j=1}^{12} \sum_{i=1}^{6} N_{j k} \frac{\left(f_{i j k}-p_{i j k}\right)^{2}}{p_{i j k}}$,

where $i$ represents the number of bins into which the RTs were separated, $j$ represents the number of conditions in the experiment (viz., six difficulty groups for both correct and error responses), $k$ represents "low" and "high" responses, $f_{i j k}$ is the observed frequency for each condition in each bin $i, p_{i j k}$ is the predicted frequency for each condition in each bin, and $N_{j k}$ is the number of observations in condition $j$ and response type $k$.
On the basis of the best-fitting model in Leite and Ratcliff (2011; viz., Model II), I parameterized the diffusion model using 16 free parameters (see Table 1 for the parameter descriptions). ${ }^{2}$ The following parameters were assumed not to change across manipulations of stimulus frequency and payoffs: boundary separation, nondecision time and its variability, three pairs of mean drift rates (one for each difficulty level), and variability in drift rate (due to the combination of stimuli of slightly different discriminability in each difficulty level). ${ }^{3}$ The starting points at the two bias levels and the variability in starting point changed between the stimulus frequency and payoff manipulations. Its scaling parameter, $s$, was set to 0.1 , as is commonly reported (e.g., Ratcliff \& Rouder, 1998).

Analogous to the specification of the diffusion model, I parameterized the two-stage model using 14 free parameters: boundary separation, nondecision time, and three pairs of mean drift rates were assumed not to change across the stimulus frequency and payoff conditions; first-stage drift rates and the switching probability for each of the two bias levels could differ between the two conditions. Because of the difference in the numbers of parameters between the two models, in what follows I present results obtained both with these differences and after reparameterizing one of the models such that the same number of free parameters were included in both models (see Appendix A for alternative comparisons).

\section{Results}

\section{Experimental effects}

In the experiment, bias was manipulated in two directions: toward "high" responses and toward "low" responses-abbreviated hereinafter as bias-high and bias-low, respectively. In each of these two levels, there were instances of correct "high" and "low" responses. I refer to the correct "high" responses in the bias-high condition and to the correct "low" responses in the bias-low condition as congruent trials (between bias level and response type); conversely, correct "low" responses in bias-high and correct "high" responses in bias-low are referred to as incongruent trials.

Consistent with previous findings, the median correct RT slowed down and accuracy decreased from congruent to incongruent trials (with respect to frequency bias) in the stimulus frequency manipulation (cf. Kirby, 1976; Laming, 1968; Luce, 1986; Posner, 1978). The effects in the payoff

\footnotetext{
${ }^{2}$ There are seven fewer parameters than in Model II of Leite and Ratcliff (2011) because I did not analyze the data from two conditions: decision cutoff and stimulus frequency with decision cutoff.

${ }^{3}$ This follows Stone's (1960) original proposal that the rate of the flow of information can be varied by changing the stimulus only, as RTs are determined by the time taken to extract information from the stimulus. I thank D. Laming for emphasizing this point during the review.
} 
condition, albeit in the same direction, were not as strong as those in the stimulus frequency condition (for other behavioral studies involving similar bias manipulations that have also shown weaker effects for payoffs than for stimulus frequency, see Maddox \& Bohil, 1998; Simen et al., 2009). ANOVA results showed that there was a significant interaction effect of bias (toward "high" responses and toward "low" responses), response type (high and low), and manipulation (stimulus frequency and payoff) on median correct RTs $-F(1,5)=$ $22.298, p=.005$ - and a nonsignificant effect on accuracy$F(1,5)=6.314, p=.054$. The boxplots in Figs. 2 and 3 illustrate the facilitatory effect on median RTs (for correct responses) and accuracy, respectively.

\section{Modeling analyses}

As is described above, the models were fit to each participant's data set, and the correct- and error-RT distributions were each approximated by five quantiles. These quantiles, in turn, divided the RTs into six bins. There were 24 conditions (viz., 2 bias levels $\times 2$ manipulation levels $\times 3$ difficulty levels $\times 2$ response types) and, hence, 264 degrees of freedom in each participant's data set: 24 conditions $\times(12$ bins -1$)$. The results in Table 2 show that the diffusion model produced smaller values than the two-stage model, but also show that boundary separation, nondecision time, and the stimulus-based drift rate estimates were very well matched across models (for the parameter estimates for each individual participant, see Appendices B and C).

For example, boundary separation $(a)$ is approximately twice as large in the two-stage model as in the diffusion model (this difference was expected, due to the different scalings of the models), and drift rates move toward zero as difficulty increases (from $\nu_{1}$ to $\nu_{3}$ and from $\nu_{6}$ to $\nu_{4}$ ). Thus, interpretation of the behavioral effects of stimulus frequency and payoffs was performed on the basis of the parameters that varied between the stimulus frequency and payoff structure trials (viz., starting point and starting point variability, in the diffusion model, and probability of switching from Stage 1 to Stage 2 and initial drift rate, in the two-stage model).
Fig. 2 Facilitatory effect on median correct RTs in Leite and Ratcliff (2011, Exp. 2). Arrows in the boxplots show an increase in median RT from congruent trials (i.e., bias to "high" responses when "high" was the correct response and bias to "low" responses when "low" was the correct response) to incongruent trials (i.e., bias to "low" responses when "high" was the correct response and bias to "high" responses when "low" was the correct response). Such an increase is more pronounced in the stimulus frequency condition than in the payoff condition

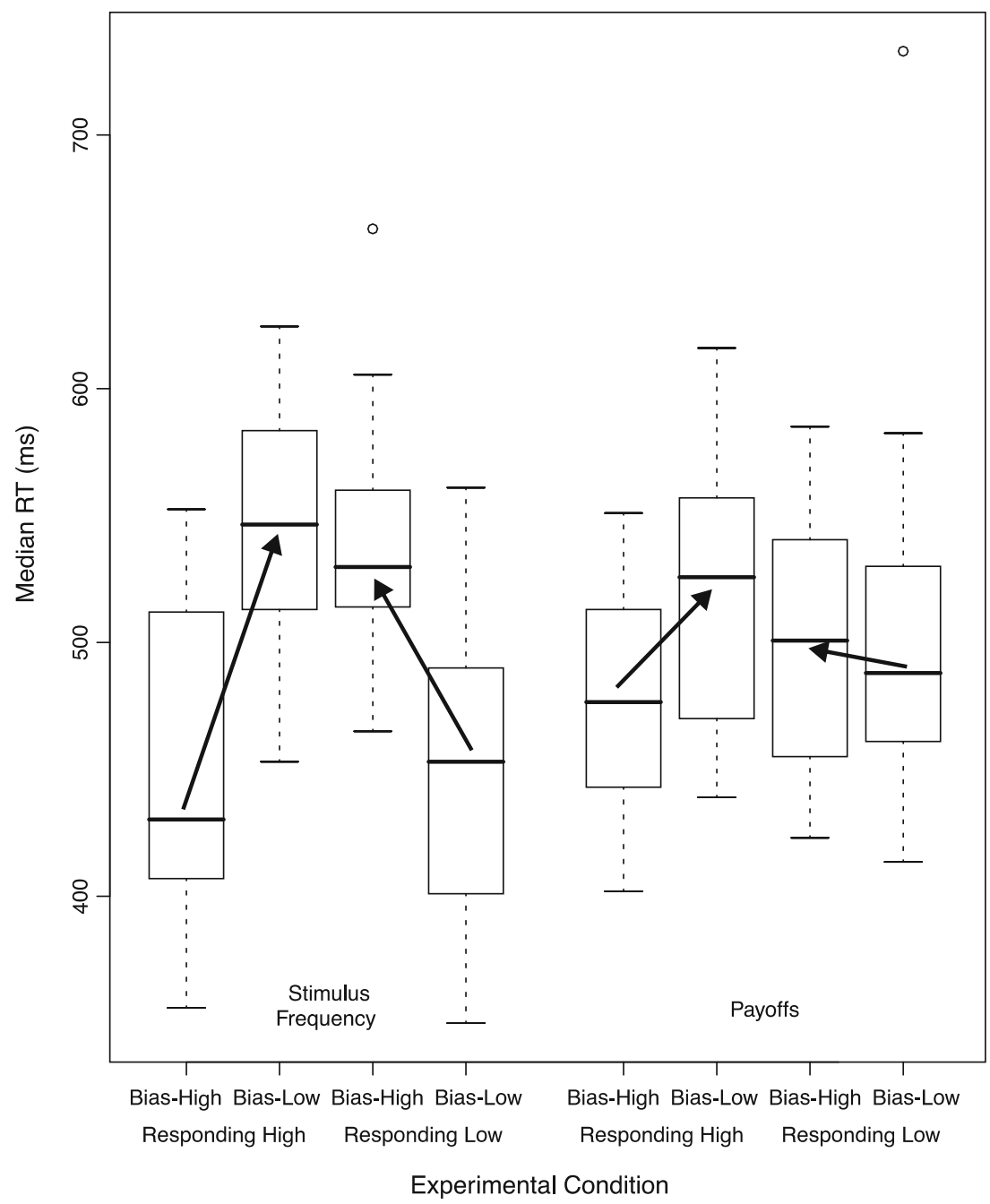


Fig. 3 Facilitatory effect on accuracy in Leite and Ratcliff (2011, Exp. 2). Arrows in the boxplots show a decrease in accuracy from congruent trials (i.e., bias to "high" responses when "high" was the correct response and bias to "low" responses when "low" was the correct response) to incongruent trials (i.e., bias to "low" responses when "high" was the correct response and bias to "high" responses when "low" was the correct response). Such a decrease is more pronounced in the stimulus frequency condition than in the payoff condition

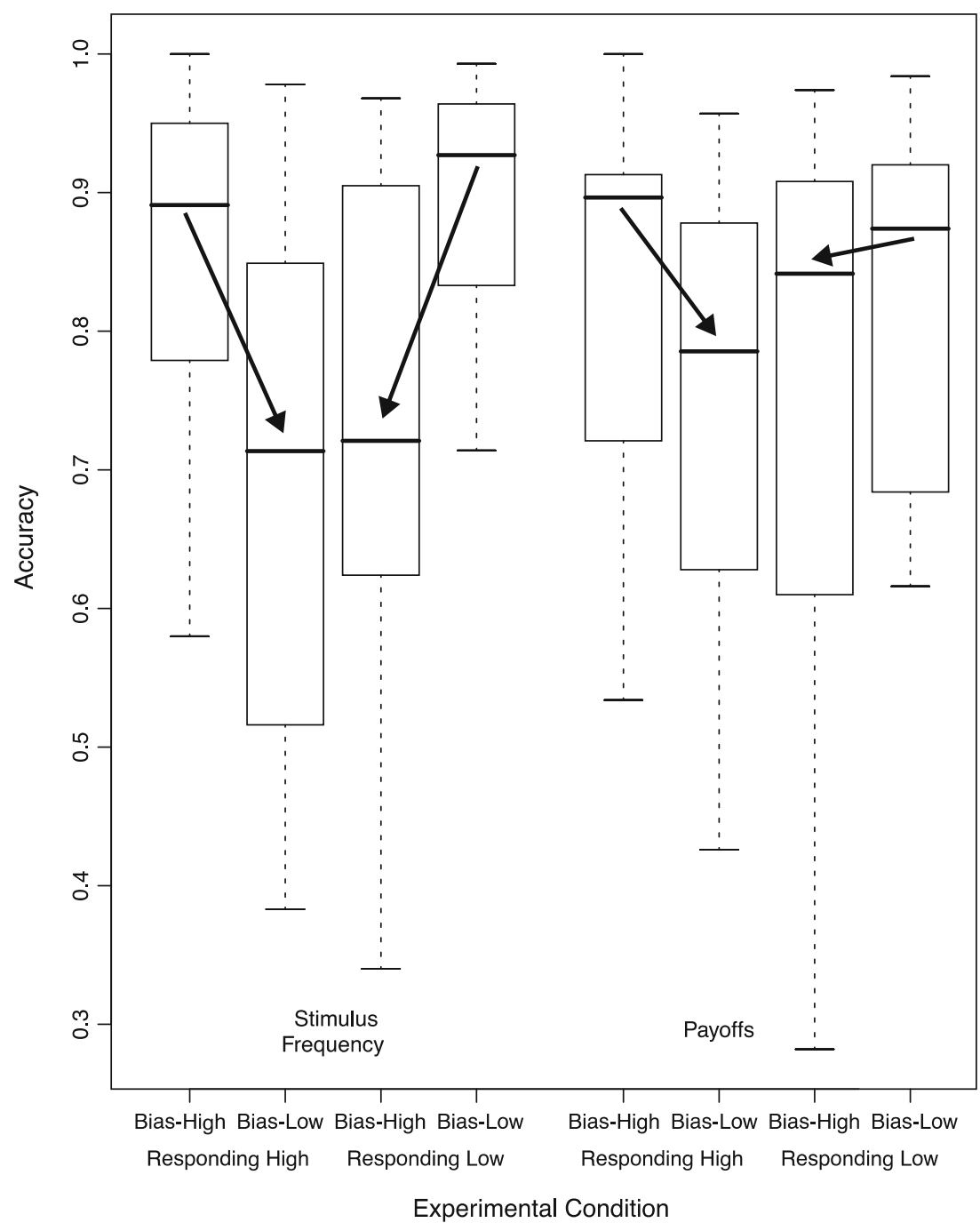

In the diffusion model, stimulus frequency effects between bias conditions were accounted for by an average increase in starting point of $94 \%$ (Table $2, z_{\mathrm{h}} / z_{1}-1$ ), whereas payoff effects between bias conditions were accounted for by an average increase in starting point of $19 \%$. In the two-stage model, stimulus frequency effects between bias conditions were accounted for by an average difference in the drift rate associated with processing the frequency information of 0.230 units (Table $2, \rho_{\mathrm{h}}-\rho_{\mathrm{l}}$ ), with a $3.8 \%$ chance of switching to the processing of the stimulus information at each step of the accumulation process; payoff effects between bias conditions were accounted for by an average change in starting point of
Table 2 Mean parameter estimates

$a=$ boundary; $T_{\mathrm{er}}=$ nondecision time (in seconds); $s_{t}=$ range of variability in $T_{\mathrm{er}} ; z_{r}=$ starting point at the $r$ bias level: $1=$ low, $\mathrm{h}=$ high; $s_{z}=$ range of variability in starting point; $\eta=$ variability in drift rate; $\nu_{n}=$ drift rate for the $n$ stimulus subgroup; $\mathrm{swt}=$ switching probability; $\rho_{r}=$ bias drift rate for the $r$ bias level

\begin{tabular}{|c|c|c|c|c|c|c|c|c|c|}
\hline \multicolumn{10}{|c|}{ Diffusion Model } \\
\hline$a$ & $T_{\mathrm{er}}$ & $s_{t}$ & $\eta$ & $\nu_{1}$ & $\nu_{2}$ & $\nu_{3}$ & $\nu_{4}$ & $\nu_{5}$ & $\nu_{6}$ \\
\hline 0.108 & 0.351 & 0.181 & 0.158 & -0359 & -0.262 & -0.094 & 0.066 & 0.227 & 0.324 \\
\hline \multicolumn{4}{|c|}{ Stimulus Frequency } & \multicolumn{6}{|l|}{ Payoffs } \\
\hline$z_{1}$ & $z_{\mathrm{h}}$ & $s_{z}$ & & $z_{1}$ & $z_{\mathrm{h}}$ & $s_{z}$ & & $\chi^{2}$ & \\
\hline 0.036 & 0.070 & 0.039 & & 0.052 & 0.062 & 0.030 & & 593.5 & \\
\hline \multicolumn{10}{|c|}{ Two-Stage Model } \\
\hline$a$ & $T_{\mathrm{er}}$ & & & $\nu_{1}$ & $\nu_{2}$ & $\nu_{3}$ & $\nu_{4}$ & $\nu_{5}$ & $\nu_{6}$ \\
\hline 0.212 & 0.295 & & & -0.071 & -0.051 & -0.021 & 0.019 & 0.046 & 0.065 \\
\hline \multicolumn{4}{|c|}{ Stimulus Frequency } & \multicolumn{6}{|l|}{ Payoffs } \\
\hline swt & $\rho_{1}$ & $\rho_{\mathrm{h}}$ & & swt & $\rho_{1}$ & $\rho_{\mathrm{h}}$ & & $\chi^{2}$ & \\
\hline 0.038 & -0.111 & 0.119 & & 0.050 & -0.116 & 0.096 & & $1,559.0$ & \\
\hline
\end{tabular}


0.212 units under a $4.9 \%$ chance of switching to the processing of the stimulus information at each step of the accumulation process.

To illustrate the fits, I plotted the data and model predictions (averaged across all six participants) in the quantile-probability functions in Figs. 4 (frequency manipulation) and 5 (payoff manipulation). The plots in those figures show information about all of the data: the proportions of correct and error responses and the shapes of the RT distributions for correct and error responses. They show that the diffusion model and the two-stage model performed similarly well in capturing the data pattern, especially for correct responses. This type of mimicking between competitive models has been reported and discussed elsewhere (e.g., Ashby \& Townsend, 1980; Leite \& Ratcliff, 2010; Ratcliff \& Smith, 2004), although the models I tested did not mimic one another in all instances, particularly not among error responses or at the extreme ends of the correctRT distributions (i.e., .1 and .9 quantiles).

In the stimulus frequency manipulation (Fig. 4), the twostage model overestimated the leading edge (i.e., .1 quantiles) of the correct RT distributions in congruent trials (top-left and bottom-right panels), whereas the diffusion model's prediction matched the data very closely. In incongruent trials (top-right and bottom-left panels), there were small misses in accuracy by both models and overestimation of the leading edge of the error-RT distributions by the two-stage model.

Similarly, in the payoff manipulation (Fig. 5), the two-stage model overestimated the leading edge of the correct-RT distributions in congruent trials. When the payoff structure favored "high" responses, the diffusion model's prediction matched the data very closely; when the payoff structure favored "low" responses, the diffusion model underestimated the leading edge of the correct RT distributions.

To examine the extent to which the two models produced similar accounts of the same cognitive processes, I simulated the two-stage model using the values in Table 2 and producing 20,000 iterations in each condition. The results of this simulation showed that $\rho_{\mathrm{l}}=-0.109$ and $\rho_{\mathrm{h}}=0.117$ (stimulus frequency) produced average starting points for the second stage equal to 0.086 and 0.112 , equivalent to $41 \%$ and $54 \%$ of the distance between the "low" and "high" boundaries, respectively. In contrast, the diffusion model estimates were
Fig. 4 Quantile-probability plots for data and the predictions from both the diffusion model and the twostage model in the stimulus frequency manipulation. Quantile-RT data points, averaged across participants, are plotted in ascending order (from .1 to .9 , in increments of .2) in each column of five circles. In each of the four panels, reading from left to right, there are three such columns across error responses, followed by three columns across correct responses, making up the six difficulty conditions (for asterisk counts of 36-40, 41-45, 46$50,51-55,56-60$, and 61-65)

\section{Stimulus Frequency}
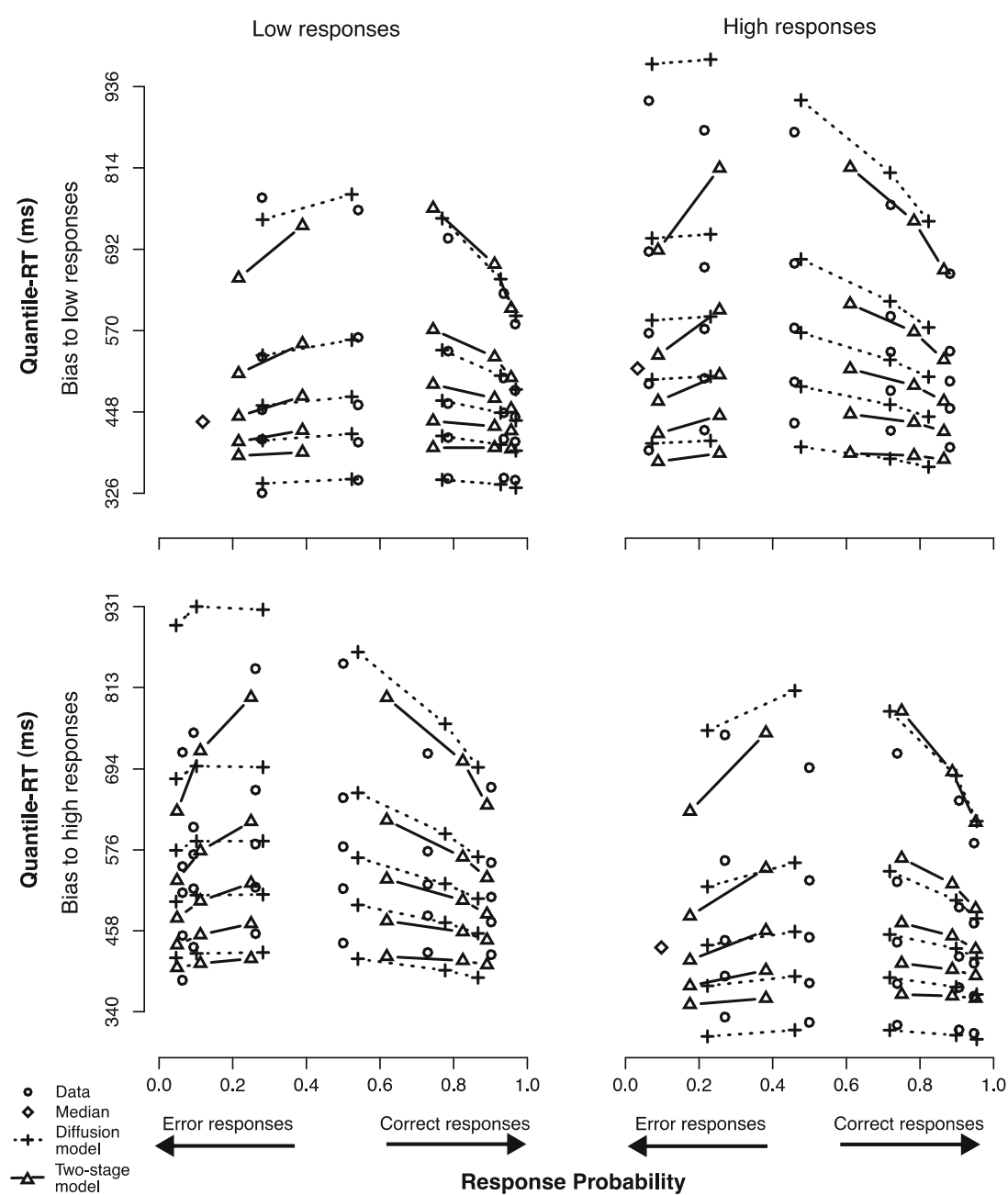

Response Probability

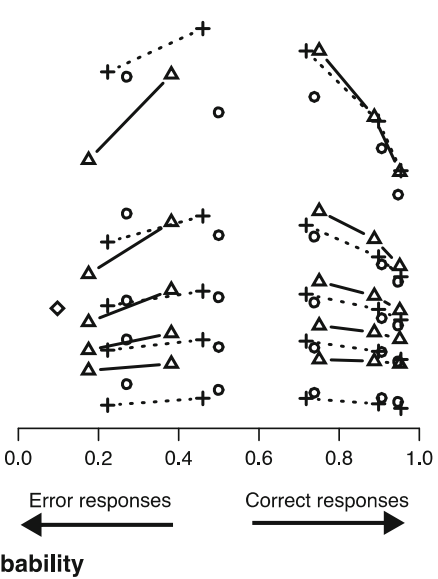


Fig. 5 Quantile-probability plots for data and the predictions from both models (akin to Fig. 4) in the payoff structure manipulation
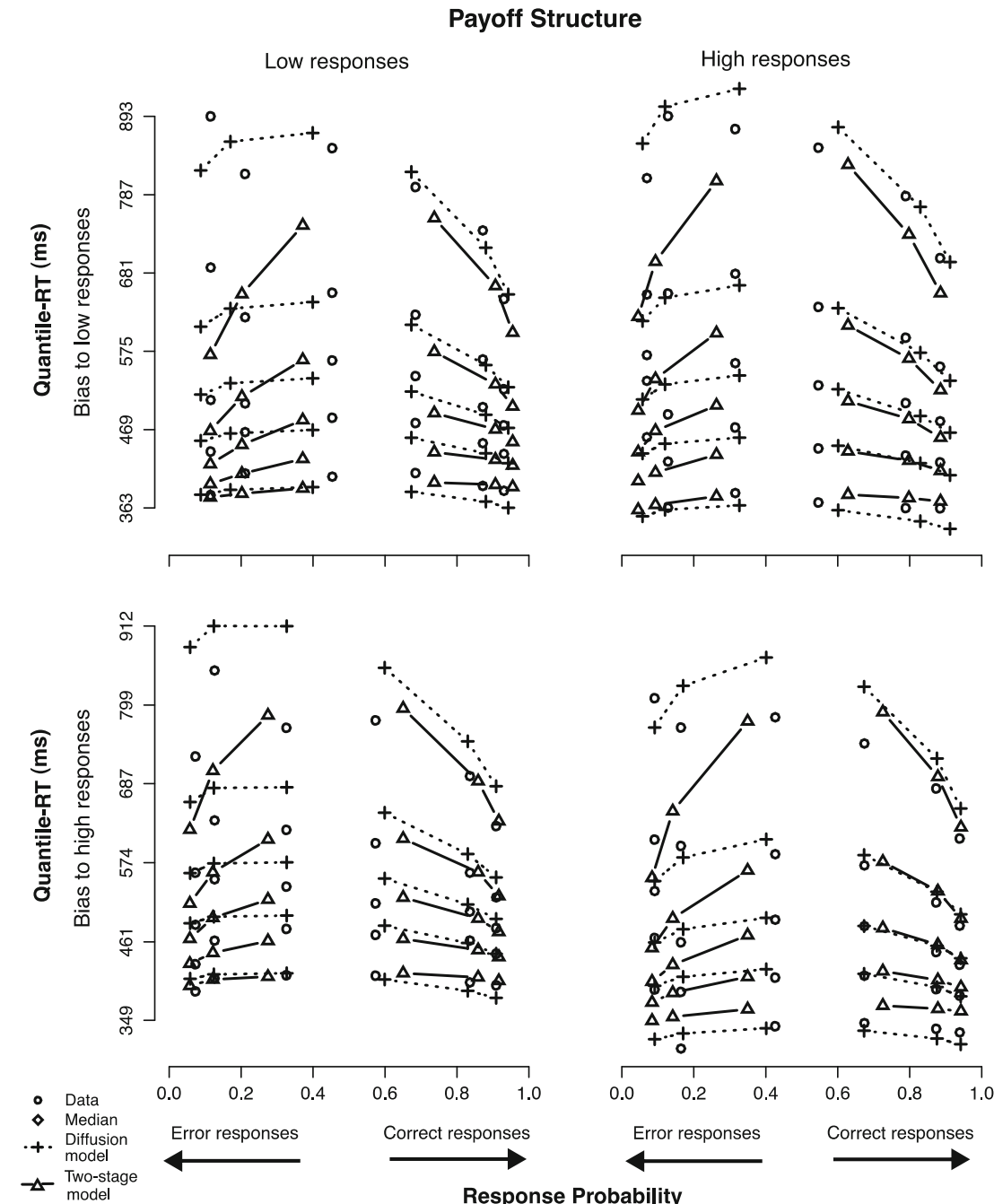

Response Probability

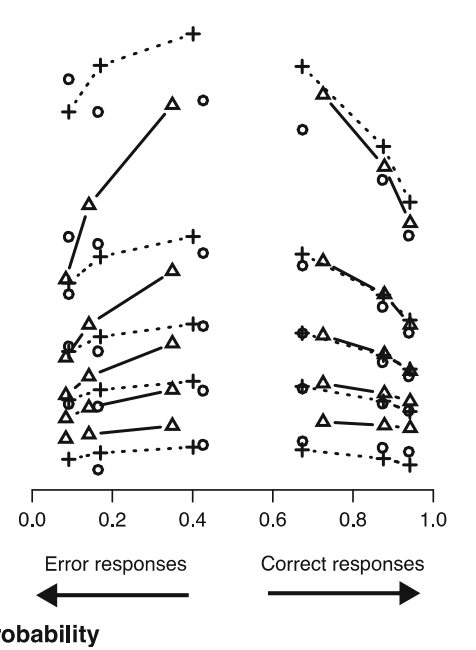

equivalent to $33 \%$ and $65 \%$ of the distance between the two boundaries, respectively. Figure 6 illustrates how similarly the effective variability in starting point of the second-stage process in the two-stage model was to the formal variability in starting point in the diffusion model.

Because the models did not have the same number of free parameters and yet produced similar accounts of the data, the performance comparison could potentially be enhanced to disambiguate the influence of the number of parameters with the use of, for example, the Akaike information criterion (AIC; Akaike, 1974). Because of the magnitude of the difference in $\chi^{2}$ values for the two models, however, a reanalysis with AIC would not suffice to determine whether less flexibility or major assumptions regarding the perceptual decisions were hindering the two-stage model's performance. Rather, in what follows, I present a reanalysis in which I reparameterized the two-stage model to include two sources of across-trial variability with which the diffusion model was fit.

In the analyses above, it was evidenced that the two-stage model might have been at a disadvantage fitting slow error responses because it was not parameterized with cross-trial variability in stimulus-driven drift rate, which has been shown to help accommodate such responses (cf. Van Zandt \& Ratcliff, 1995). Consequently, here I present model-fitting results (minimized via Eq. 1) in which both models assumed that nondecision time and stimulus-driven drift rates varied across trials. Hence, the potential disadvantage to the two-stage model relative to the diffusion model was eliminated, and the two models were matched in their numbers of parameters, such that a $\chi^{2}$ comparison was straightforward.

Adding variability in nondecision time and in stimulusdriven drift rates improved the individual fits of the twostage model to every participant (see Appendix D). On average, the parameter estimates were similar to the parameters estimates produced without the added variability, but the $\chi^{2}$ values were closer to the values produced by the diffusion model (Table 3). Thus, the boundary separation, nondecision time, and stimulus-based drift rate estimates were very well matched across the models, with the latter moving toward zero as difficulty increased (from $\nu_{1}$ to $\nu_{3}$ and from $\nu_{6}$ to $\nu_{4}$ ).

To illustrate the fits, I plotted the data and model predictions (averaged across all six participants) in quantile- 
Fig. 6 Comparison of startingpoint distributions in the two models. The plot shows the ratio between starting-point and boundary estimates (normalizing the starting-point estimates) as a function of the ratio's cumulative density, to illustrate how the starting points were distributed across trials. Solid lines represent the diffusion model, and dashed lines represent the two-stage model
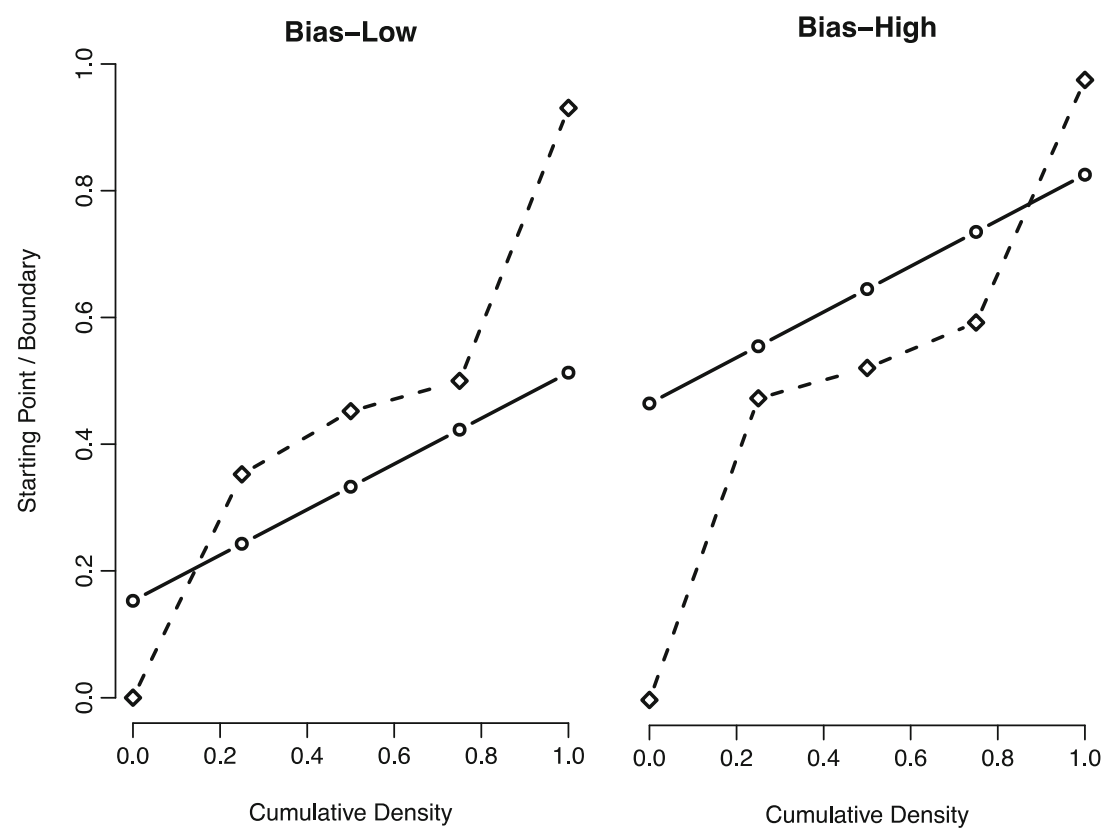

probability functions in Figs. 7 (frequency manipulation) and 8 (payoff manipulation). Consistent with the $\chi^{2}$ results summarized in Table 3, the fits show minimal differences between the model predictions, most noticeably in underestimations of error response probability (stimulus frequency condition) and underestimations of error RTs in the two-stage model relative to the diffusion model. In comparison to Figs. 4 and 5, and consistent with the large improvement in $\chi^{2}$ values between Tables 2 and 3, it is evident that the added variability parameters produced better fits to the data.

Once again, to examine the extents to which the two models produced similar accounts of the same cognitive processes, I simulated the two-stage model using the values in Table 3 and producing 20,000 iterations in each condition. The results of this simulation replicated the previous results: $\rho_{1}=-0.115$ and $\rho_{\mathrm{h}}=0.125$ (stimulus frequency) produced average starting points for the second stage equal to 0.097 and 0.123 , equivalent to $43 \%$ and $54 \%$ of the distance between the low and high boundaries, respectively. Because this difference in starting points across conditions mimics that in the diffusion model (viz., $33 \%$ and $65 \%$ of the same distance, respectively), I compared how much variability in starting points the two-stage model had built in, relative to the diffusion model (Fig. 9). Figure 9 is nearly identical to Fig. 6 because the addition of variability in nondecision time and in stimulus-driven drift rate does not affect the accumulation of information during the first stage (based on the attribute information).

\section{Discussion}

Although payoffs and prior probabilities are known to bias choices in near-threshold discrimination tasks, Leite and Ratcliff (2011) showed that these manipulations of stimulus frequency and payoffs required different treatments of the starting point of the decision process in the

Table 3 Mean parameter estimates: Matching variability parameters

\begin{tabular}{|c|c|c|c|c|c|c|c|c|c|c|}
\hline Model & $a$ & $T_{\mathrm{er}}$ & $s_{t}$ & $\eta$ & $\nu_{1}$ & $\nu_{2}$ & $\nu_{3}$ & $\nu_{4}$ & $\nu_{5}$ & $\nu_{6}$ \\
\hline $\mathrm{DM}$ & 0.108 & 0.351 & 0.181 & 0.158 & -0359 & -0.262 & -0.094 & 0.066 & 0.227 & 0.324 \\
\hline \multirow[t]{3}{*}{$2-\mathrm{S}$} & 0.227 & 0.314 & 0.107 & 0.021 & -0.066 & -0.049 & -0.020 & 0.018 & 0.044 & 0.065 \\
\hline & \multicolumn{4}{|c|}{ Stimulus Frequency } & \multicolumn{6}{|l|}{ Payoffs } \\
\hline & $z_{1}$ & $z_{\mathrm{h}}$ & $s_{z}$ & & $z_{1}$ & $z_{\mathrm{h}}$ & $s_{z}$ & & $\chi^{2}$ & \\
\hline \multirow[t]{2}{*}{$\mathrm{DM}$} & 0.036 & 0.070 & 0.039 & & 0.052 & 0.062 & 0.030 & & 593.5 & \\
\hline & swt & $\rho_{1}$ & $\rho_{\mathrm{h}}$ & & swt & $\rho_{1}$ & $\rho_{\mathrm{h}}$ & & & \\
\hline $2-S$ & 0.041 & -0.115 & 0.125 & & 0.049 & -0.106 & 0.121 & & 803.7 & \\
\hline
\end{tabular}

$a=$ boundary; $T_{\mathrm{er}}=$ nondecision time (in seconds); $s_{t}=$ range of variability in $T_{\mathrm{er}} ; z_{r}=$ starting point at the $r$ bias level: $1=$ low, h= high; $s_{z}=$ range of variability in starting point; $\eta=$ variability in drift rate; $\nu_{n}=$ drift rate for the $n$ stimulus subgroup; swt $=$ switching probability; $\rho_{r}=$ bias drift rate for the $r$ bias level. DM refers to the diffusion model; 2-S refers to the two-stage model 
Fig. 7 Quantile-probability plots for data and the predictions from both the diffusion model and the twostage model (with added variability parameters) in the stimulus frequency manipulation (cf. Fig. 4)

\section{Stimulus Frequency}
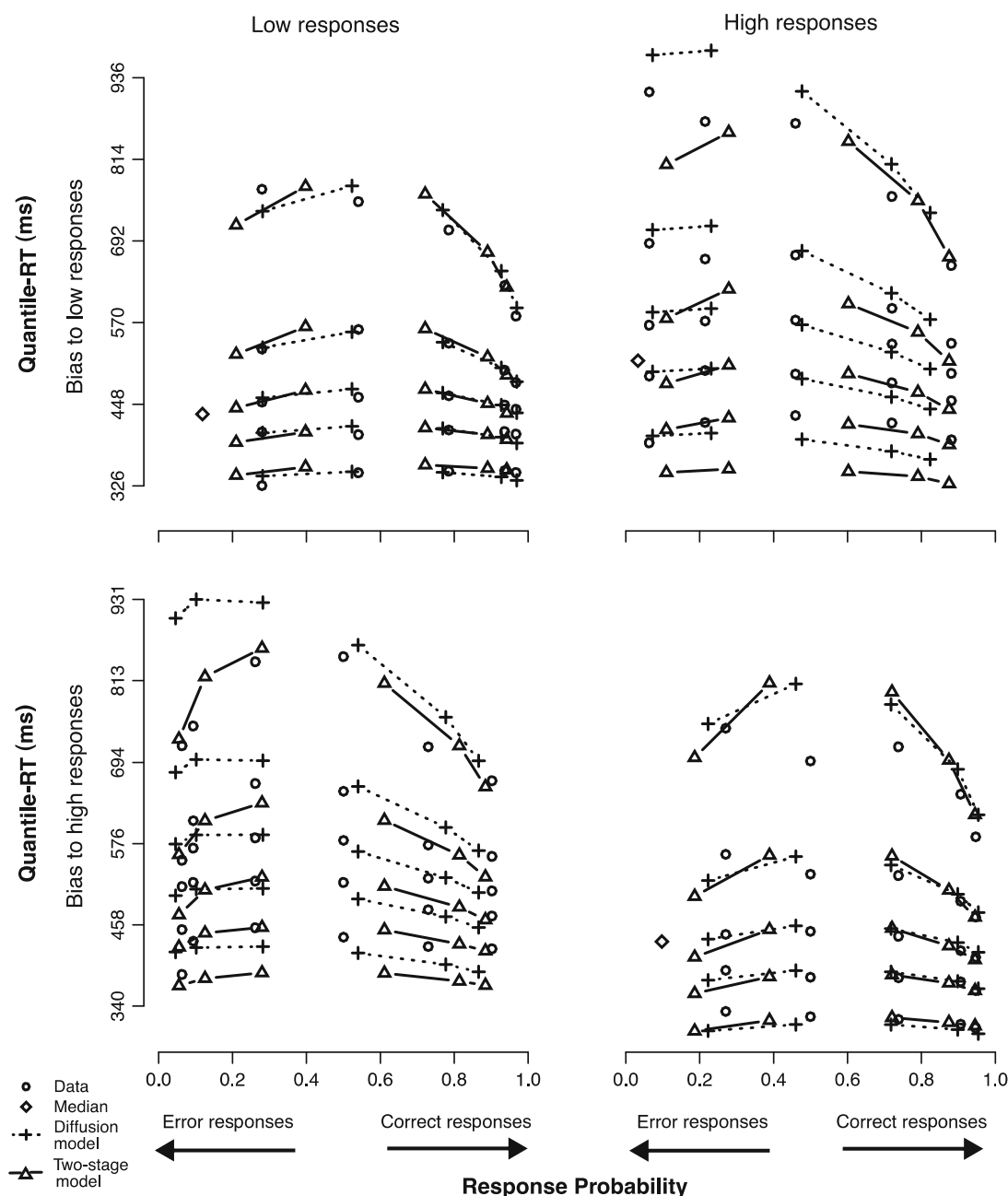

Response Probability diffusion model. Such weaker effects for the payoff than for the prior-probability manipulation have also been reported and discussed elsewhere (e.g., Maddox \& Bohil, 1998; Simen et al., 2009), suggesting a systematic cause for it (see also Mulder, Wagenmakers, Ratcliff, Boekel, \& Forstmann, 2012). Following Diederich and Busemeyer (2006), processing the information from the payoff may be a contributing factor. Thus, my objective in the present study was to test the relative merits of a single-stage diffusion model (Ratcliff, 1978) and a two-stage diffusion model (Diederich \& Busemeyer, 2006) in accounting for manipulations of stimulus frequency and payoffs. From this examination, I learned the following two main points.

One, the two-stage model produced better fits to perceptual-decision data by incorporating cross-trial variability in the parameters. Specifically, I added a parameter modeling cross-trial variability in the stimulusdriven drift rate, assuming that participants did not extract identical perceptual information from similar stimuli across trials, and in nondecision time, assuming that participants did not take identical time to encode the stimulus information across trials (both assumptions are consistent with Van Zandt \& Ratcliff, 1995). On average, the addition of these two parameters to the formulation in Diederich and Busemeyer (2006) approximately halved the resulting $\chi^{2}$ values associated with the fits to the data (cf. the two-stage $\chi^{2} \mathrm{~s}$ in Table 2 and Table 3).

Two, a comparison between these two diffusionprocess models showed more similarities than differences. In spite of their formal differences (viz., singlestage and time-homogeneous in the diffusion model vs. two-stage and time-inhomogeneous in the two-stage model), their similar architectures effectively produce nearly equivalent mathematical predictions. That is, a brief initial spurt of activity in the decision process (driven by information about the payoffs or stimulus frequency) that is later definitively overtaken by the processing of the stimulus evidence is similar to oneshot processing of the stimulus that starts closer to the boundary associated with the most frequent or valued response. Put differently, having a two-stage process of 
Fig. 8 Quantile-probability plots for data and the predictions from both the diffusion model and the twostage model (with added variability parameters) in the payoff structure manipulation (cf.

Fig. 5)

\section{Payoff Structure}
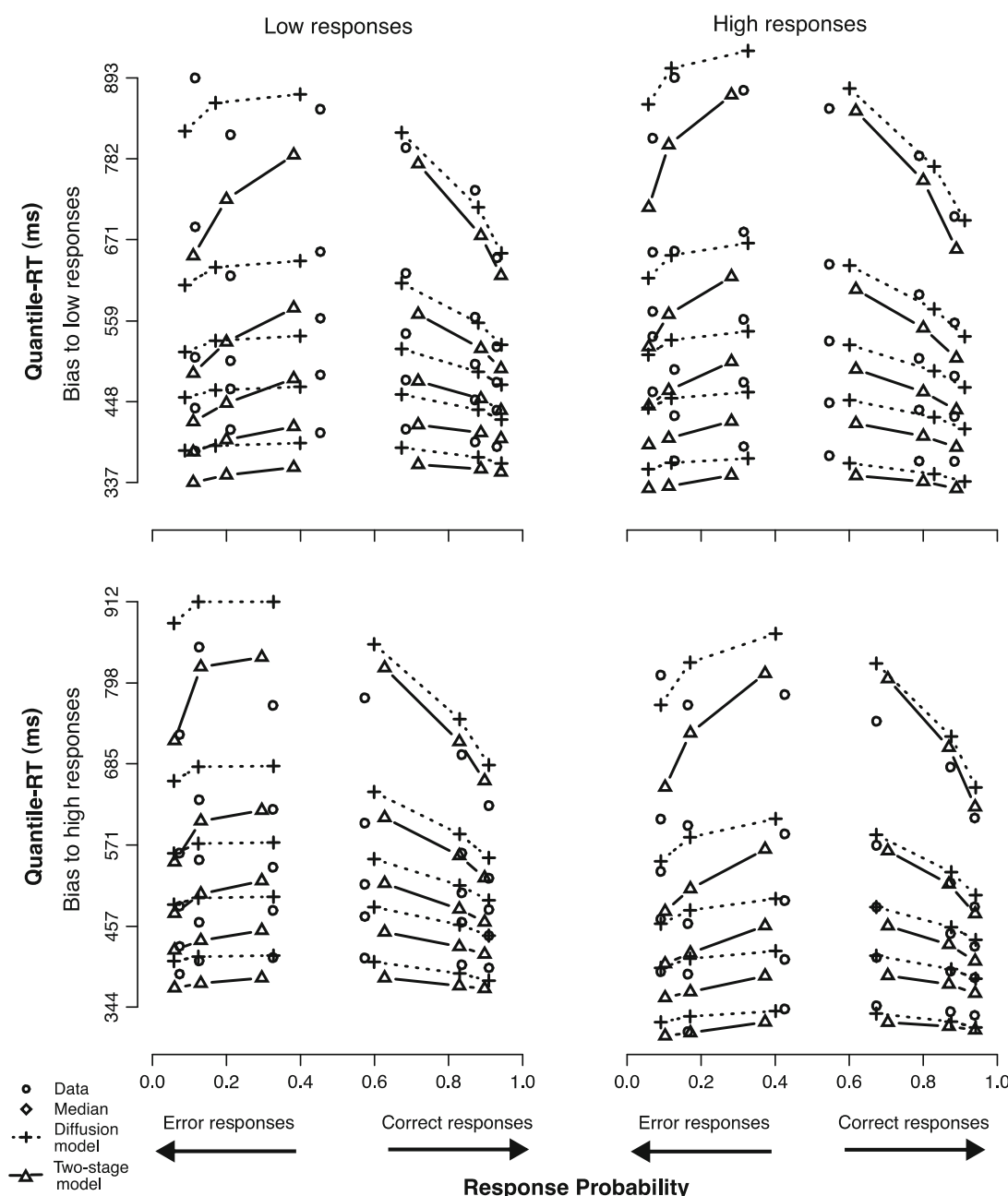

Response Probability

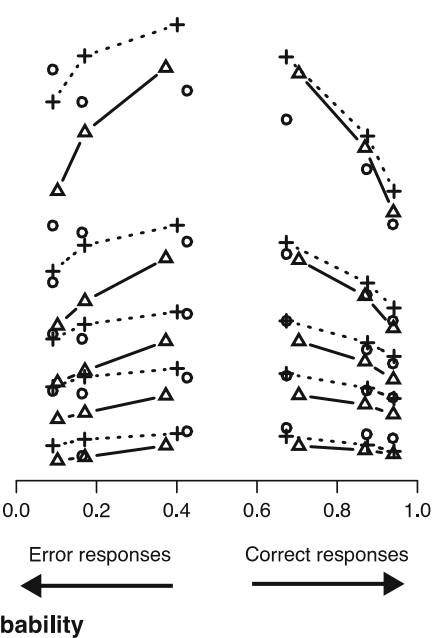

evidence accumulation is similar to having variability plus shift in starting point in a single-stage process of accumulation (see the comparisons in Figs. 6 and 9).

This last point differs from the findings of Diederich and Busemeyer (2006), but the two studies differed in their methods and aims. In the present study, there was no test of a drift-rate-change assumption, because Leite and Ratcliff (2011) showed that changes in both stimulus frequency and payoffs affect starting point, not drift rate. Moreover, this study addressed differences in the entire RT distribution, rather than only in mean RTs, and the task examined by Diederich and Busemeyer was much different than the task I examined here.

As a consequence of the observed nearly equivalent predictions and performance in fitting the data by the two models (when parameterized analogously), I find that a choice between the two models may not represent a decision between two different possibilities - as it might appear initially. At the least, a cognitive scientist selecting one of the two models might want to consider factors such as the assumptions of each model and the interpretation of their parameter estimates, rather than model performance only.

For example, in the diffusion model, assuming that knowledge of stimulus frequency maps onto the starting point of accumulation derives from interpreting the relative stimulus frequencies as prior information, whereas the decision process associated with the random walk is driven by the information available in the stimulus in each trial. Likewise, knowledge of payoffs (not dependent on RT) map naturally onto the boundaries of the accumulation process, whose adjustments are equivalent to adjustments of the starting point. The resulting estimates of starting point can then be interpreted in terms of the amount of bias toward a response that the specific payoff structure or stimulus frequency induced (e.g., Leite \& Ratcliff, 2011). In the two-stage model, knowledge of payoffs is mapped onto the initial drift rate in the accumulation process because the random walk is assumed to initiate before the stimulus information is processed (although it is available). In effect, however, these initial drift rates produce variability in 
Fig. 9 Comparison of startingpoint distributions in the two models. The plot shows the ratio between starting-point and boundary estimates (normalizing the starting-point estimates) as a function of the ratio's cumulative density, to illustrate how the starting points were distributed across trials. Solid lines represent the diffusion model, and dashed lines represent the two-stage model
Bias-Low

Bias-High

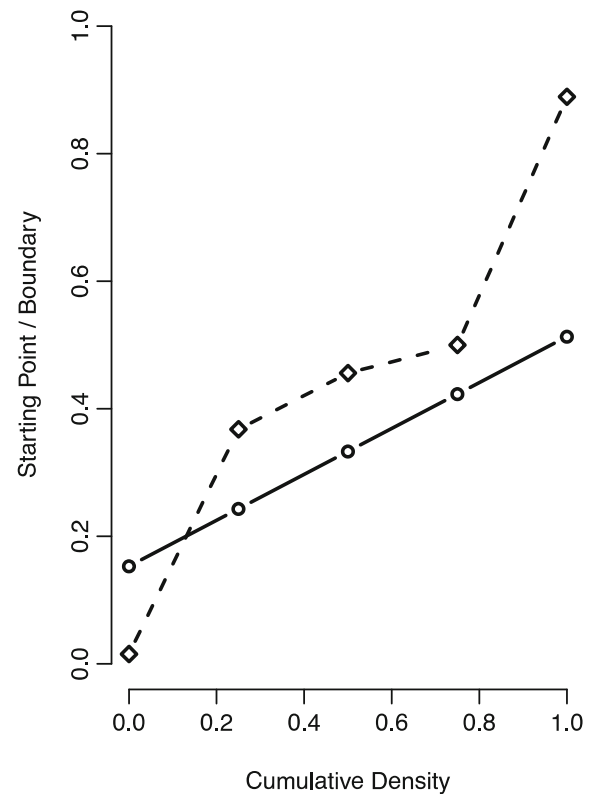

the starting point of the second stage and, on occasion, ${ }^{4}$ are the only drift rates driving the process of accumulation until a decision is reached. Thus, I find that interpreting the switching-probability estimate and the estimates of the two types of drift rate may be less trivial than interpreting starting point differences across conditions.

Author note This article reanalyzes data reported in Leite and Ratcliff (2011). I thank Adele Diederich and Roger Ratcliff for invaluable comments during early stages of this project.

\section{Appendix A: Model fitting via an alternative object function}

As it is typically implemented, the two-stage model is minimized over a different object function than Eq. 1, as will now be described (cf. Diederich \& Busemeyer, 2006). The response probabilities are fit by minimizing the function

$g_{\text {prob }}=\sum_{k=1}^{2} \sum_{j=1}^{12}\left(\frac{f_{j k}-p_{j k}}{s e\left(p_{j k}\right)}\right)^{2}$

where $j$ and $k$ stand as in Eq. 1, $f_{j k}$ is the observed relative frequency for each condition, $p_{j k}$ is the predicted probability, and

\footnotetext{
${ }^{4}$ In the same simulation routine presented on p. 20 , for example, the bias-driven drift rate was never replaced by a stimulus-driven drift rate in, on average, $1.3 \%$ of the 20,000 stimulated accumulation paths.
}

$s e\left(p_{j k}\right)=\sqrt{\frac{p_{j k}\left(1-p_{j k}\right)}{N_{j k}}}$,

the standard error with $N_{j k}$ as the number of observations in each condition and response category. The RTs are fit by minimizing the function

$g_{R T}=\sum_{k=1}^{2} \sum_{j=1}^{12} \sum_{q=1}^{5} N_{j k}\left(R T(o b s)_{q j k}-R T(\text { pred })_{q j k}\right)^{2}$,

where $j$ and $k$ stand as in Eq. 1, $q$ represents the five quantiles that marked the six bins in Eq. 1, $N_{j k}$ is equal to the number of observations in each condition and response category, and RT (obs) and RT(pred) are the observed and predicted choice RTs at the five quantiles given above, respectively. The overall objective function, $g=g_{\text {prob }}+g_{\mathrm{RT}}$, is minimized by using the MATLAB routine "fminsearch" (based on the simplex method; Lagarias, Reeds, Wright, \& Wright, 1998).

In addition to fitting both models via Eq. 1, I also fitted them via objective function $g$ to ensure that no model had an advantage over the other on the basis of the fitting method. ${ }^{5}$ Tables 4 and 5 show the parameter estimates of the diffusion model and of the two-stage model for each individual participant, respectively, fitted via the two-stage model's $g$.

\footnotetext{
${ }^{5}$ Another method, involving the quantile maximum probability estimate (QMPE) method (Cousineau, Brown, \& Heathcote, 2004; Heathcote, Brown, \& Cousineau, 2004), was also tested. Its results are not reported because they were consistent with the present results, and because that method introduced a potential source of error by assuming that the RT distributions were accurately represented by ex-Gaussian distributions.
} 
Table 4 Diffusion model parameter estimates $(g)$ $a=$ boundary; $T_{\mathrm{er}}=$ nondecision time (in seconds); $s_{t}=$ range of variability in $T_{\mathrm{er}} ; \eta=$ variability in drift rate; $\nu_{n}=$ drift rate for the $n$ stimulus subgroup; $z=$ starting point at trials with bias toward "low" or "high" responses; $s_{z}=$ range of variability in starting point; $g$ = goodness-of-fit measure

Table 5 Two-stage model parameter estimates $(g)$

$a=$ boundary; $T_{\mathrm{er}}=$ nondecision time (in seconds); $\nu_{n}=$ drift rate for the $n$ stimulus subgroup; swt $=$ probability of switching from Stage 1 to Stage 2; $\rho=$ drift rate at Stage 1 for trials with bias toward "low" or "high" responses; $g$ = goodness-of-fit measure.

\begin{tabular}{lllllllllll}
\hline Part. & $a$ & $T_{\text {er }}$ & $s_{t}$ & $\eta$ & $\nu_{1}$ & $\nu_{2}$ & $\nu_{3}$ & $\nu_{4}$ & $\nu_{5}$ & $\nu_{6}$ \\
A & 0.104 & 0.360 & 0.221 & 0.101 & -0.379 & -0.192 & -0.044 & 0.103 & 0.269 & 0.401 \\
B & 0.119 & 0.307 & 0.084 & 0.163 & -0.420 & -0.310 & -0.108 & 0.125 & 0.328 & 0.392 \\
C & 0.125 & 0.348 & 0.156 & 0.126 & -0.354 & -0.260 & -0.076 & 0.049 & 0.204 & 0.282 \\
D & 0.113 & 0.364 & 0.185 & 0.161 & -0.364 & -0.267 & -0.087 & 0.053 & 0.202 & 0.327 \\
E & 0.100 & 0.411 & 0.155 & 0.127 & -0.317 & -0.212 & -0.078 & 0.067 & 0.217 & 0.306 \\
F & 0.092 & 0.322 & 0.134 & 0.110 & -0.345 & -0.304 & -0.128 & 0.012 & 0.191 & 0.295 \\
Mean & 0.109 & 0.352 & 0.156 & 0.131 & -0.363 & -0.258 & -0.087 & 0.068 & 0.235 & 0.334 \\
& & Stimulus Frequency & & Payoffs & & & & \\
Part. & & $z_{1}$ & $z_{\mathrm{h}}$ & $S_{z}$ & & $z_{1}$ & $z_{\mathrm{h}}$ & $S_{z}$ & & $g$ \\
A & & 0.023 & 0.077 & 0.010 & & 0.024 & 0.080 & 0.006 & & 77.0 \\
B & & 0.029 & 0.091 & 0.004 & & 0.059 & 0.059 & 0.083 & & 102.1 \\
C & & 0.049 & 0.086 & 0.096 & & 0.067 & 0.068 & 0.015 & & 84.2 \\
D & & 0.044 & 0.055 & 0.088 & & 0.053 & 0.059 & 0.084 & & 40.1 \\
E & & 0.038 & 0.059 & 0.000 & & 0.045 & 0.053 & 0.078 & & 100.2 \\
F & & 0.036 & 0.057 & 0.000 & & 0.048 & 0.048 & 0.051 & & 56.8 \\
Mean & & 0.036 & 0.071 & 0.033 & & 0.049 & 0.061 & 0.053 & & 76.7 \\
\hline
\end{tabular}

\begin{tabular}{lllllllll}
\hline Part. & $a$ & $T_{\text {er }}$ & $\nu_{1}$ & $\nu_{2}$ & $\nu_{3}$ & $\nu_{4}$ & $\nu_{5}$ & $\nu_{6}$ \\
A & 0.204 & 0.361 & -0.086 & -0.054 & -0.023 & 0.024 & 0.049 & 0.066 \\
B & 0.219 & 0.303 & -0.071 & -0.050 & -0.020 & 0.020 & 0.053 & 0.073 \\
C & 0.215 & 0.380 & -0.062 & -0.049 & -0.019 & 0.019 & 0.047 & 0.064 \\
D & 0.204 & 0.369 & -0.065 & -0.046 & -0.021 & 0.022 & 0.032 & 0.051 \\
E & 0.230 & 0.363 & -0.063 & -0.046 & -0.019 & 0.020 & 0.042 & 0.067 \\
F & 0.183 & 0.332 & -0.076 & -0.061 & -0.022 & 0.011 & 0.039 & 0.067 \\
Mean & 0.209 & 0.351 & -0.070 & -0.051 & -0.021 & 0.019 & 0.044 & 0.065 \\
& Stimulus & Frequency & & Payoffs & & & & \\
Part. & $z_{1}$ & $z_{\mathrm{h}}$ & $S_{z}$ & $z_{1}$ & $z_{\mathrm{h}}$ & $S_{z}$ & & $g$ \\
A & 0.013 & -0.094 & 0.141 & 0.015 & -0.139 & 0.098 & & 282.6 \\
B & 0.040 & -0.104 & 0.108 & 0.054 & -0.103 & 0.097 & & 234.0 \\
C & 0.016 & -0.114 & 0.116 & 0.059 & -0.126 & 0.113 & & 235.6 \\
D & 0.062 & -0.125 & 0.115 & 0.053 & -0.101 & 0.092 & & 146.3 \\
E & 0.045 & -0.114 & 0.104 & 0.050 & -0.109 & 0.102 & & 228.9 \\
F & 0.053 & -0.105 & 0.120 & 0.062 & -0.115 & 0.068 & & 93.0 \\
Mean & 0.038 & -0.109 & 0.117 & 0.049 & -0.115 & 0.095 & & 203.4 \\
\hline
\end{tabular}




\section{Appendix B}

Table 6 Diffusion model parameter estimates

$a=$ boundary; $T_{\mathrm{er}}=$ nondecision time (in seconds); $s_{t}=$ range of variability in $T_{\mathrm{er}} ; \eta=$ variability in drift rate; $\nu_{n}=$ drift rate for the $n$ stimulus subgroup; $z=$ starting point at trials with bias toward "low" or "high" responses; $s_{z}=$ range of variability in starting point

\section{Appendix C}

Table 7 Two-stage model parameter estimates

$a=$ boundary; $T_{\mathrm{er}}=$ nondecision time (in seconds); $\nu_{\mathrm{n}}=$ drift rate for the $n$ stimulus subgroup;

swt $=$ probability of switching from Stage 1 to Stage 2; $\rho=$ drift rate at Stage 1 for trials with bias toward "low" or "high" responses

\begin{tabular}{lllllllllll}
\hline Part. & $a$ & $T_{\text {er }}$ & $s_{t}$ & $\eta$ & $\nu_{1}$ & $\nu_{2}$ & $\nu_{3}$ & $\nu_{4}$ & $\nu_{5}$ & $\nu_{6}$ \\
A & 0.115 & 0.347 & 0.143 & 0.140 & -0.359 & -0.222 & -0.071 & 0.080 & 0.239 & 0.324 \\
B & 0.114 & 0.311 & 0.130 & 0.178 & -0.414 & -0.300 & -0.101 & 0.103 & 0.272 & 0.410 \\
C & 0.123 & 0.348 & 0.206 & 0.141 & -0.339 & -0.252 & -0.086 & 0.061 & 0.216 & 0.282 \\
D & 0.105 & 0.367 & 0.264 & 0.151 & -0.352 & -0.251 & -0.079 & 0.052 & 0.201 & 0.294 \\
E & 0.102 & 0.407 & 0.152 & 0.207 & -0.331 & -0.246 & -0.101 & 0.071 & 0.219 & 0.320 \\
F & 0.092 & 0.325 & 0.193 & 0.130 & -0.357 & -0.304 & -0.124 & 0.027 & 0.216 & 0.314 \\
& & Stimulus Frequency & & Payoffs & & & & \\
Part. & & $z_{1}$ & $z_{\mathrm{h}}$ & $S_{z}$ & & $z_{1}$ & $z_{\mathrm{h}}$ & $s_{z}$ & & $\chi^{2}$ \\
A & & 0.028 & 0.088 & 0.045 & & 0.038 & 0.089 & 0.003 & & 456.0 \\
B & 0.024 & 0.091 & 0.018 & & 0.059 & 0.059 & 0.001 & & 558.9 \\
C & 0.051 & 0.079 & 0.099 & & 0.066 & 0.069 & 0.008 & & 830.2 \\
D & 0.040 & 0.048 & 0.071 & & 0.051 & 0.054 & 0.073 & & 675.0 \\
E & 0.043 & 0.060 & 0.000 & & 0.049 & 0.054 & 0.030 & & 428.0 \\
F & 0.031 & 0.055 & 0.001 & & 0.046 & 0.049 & 0.066 & & 613.0 \\
\hline
\end{tabular}

\begin{tabular}{lllllllll}
\hline Part. & $a$ & $T_{\text {er }}$ & $\nu_{1}$ & $\nu_{2}$ & $\nu_{3}$ & $\nu_{4}$ & $\nu_{5}$ & $\nu_{6}$ \\
A & 0.210 & 0.295 & -0.087 & -0.054 & -0.024 & 0.024 & 0.051 & 0.069 \\
B & 0.216 & 0.252 & -0.072 & -0.050 & -0.020 & 0.020 & 0.055 & 0.074 \\
C & 0.221 & 0.296 & -0.062 & -0.049 & -0.019 & 0.019 & 0.050 & 0.062 \\
D & 0.208 & 0.274 & -0.065 & -0.049 & -0.021 & 0.021 & 0.034 & 0.053 \\
E & 0.231 & 0.365 & -0.063 & -0.044 & -0.019 & 0.020 & 0.043 & 0.067 \\
F & 0.183 & 0.290 & -0.076 & -0.062 & -0.022 & 0.012 & 0.041 & 0.066 \\
& Stimulus Frequency & & Payoffs & & & & \\
Part. & Swt & $\rho_{1}$ & $\rho_{\mathrm{h}}$ & swt & $\rho_{1}$ & $\rho_{\mathrm{h}}$ & & $\chi^{2}$ \\
A & 0.013 & -0.093 & 0.142 & 0.015 & -0.136 & 0.101 & & $1,722.4$ \\
B & 0.040 & -0.106 & 0.109 & 0.056 & -0.104 & 0.100 & & $1,735.5$ \\
C & 0.017 & -0.113 & 0.122 & 0.063 & -0.126 & 0.112 & & $1,896.5$ \\
D & 0.062 & -0.133 & 0.117 & 0.053 & -0.104 & 0.091 & & $2,428.2$ \\
E & 0.045 & -0.115 & 0.105 & 0.050 & -0.110 & 0.103 & & 444.4 \\
F & 0.053 & -0.107 & 0.120 & 0.061 & -0.117 & 0.069 & & $1,127.1$ \\
\hline
\end{tabular}




\section{Appendix D}

Table 8 Two-stage model (with added variability) parameter estimates $a=$ boundary; $T_{\mathrm{er}}=$ nondecision time (in seconds); $\nu_{n}=$ drift rate for the $n$ stimulus subgroup;

swt $=$ probability of switching from Stage 1 to Stage 2; $\rho=$ drift rate at Stage 1 for trials with bias toward "low" or "high" responses

\begin{tabular}{llllllllll}
\hline Part. & $a$ & $T_{\text {er }}$ & $\nu_{1}$ & $\nu_{2}$ & $\nu_{3}$ & $\nu_{4}$ & $\nu_{5}$ & $\nu_{6}$ & \\
A & 0.235 & 0.322 & -0.049 & -0.040 & -0.015 & 0.014 & 0.041 & 0.056 & \\
B & 0.219 & 0.272 & -0.071 & -0.050 & -0.020 & 0.020 & 0.054 & 0.073 & \\
C & 0.232 & 0.314 & -0.071 & -0.050 & -0.019 & 0.020 & 0.052 & 0.072 & \\
D & 0.231 & 0.308 & -0.064 & -0.052 & -0.025 & 0.026 & 0.036 & 0.047 & \\
E & 0.240 & 0.367 & -0.060 & -0.040 & -0.020 & 0.020 & 0.040 & 0.069 & \\
F & 0.202 & 0.301 & -0.079 & -0.061 & -0.020 & 0.010 & 0.039 & 0.070 & \\
& Stimulus Frequency & & Payoffs & & & & & \\
Part. & swt & $\rho_{1}$ & $\rho_{\mathrm{h}}$ & swt & $\rho_{1}$ & $\rho_{\mathrm{h}}$ & $\eta$ & $s_{t}$ & $\chi^{2}$ \\
A & 0.012 & -0.127 & 0.180 & 0.021 & -0.099 & 0.256 & 0.025 & 0.129 & 603.5 \\
B & 0.040 & -0.107 & 0.111 & 0.055 & -0.102 & 0.098 & 0.020 & 0.103 & $1,242.8$ \\
C & 0.039 & -0.104 & 0.110 & 0.055 & -0.102 & 0.098 & 0.020 & 0.101 & $1,267.5$ \\
D & 0.051 & -0.134 & 0.119 & 0.051 & -0.103 & 0.102 & 0.020 & 0.104 & 877.9 \\
E & 0.050 & -0.109 & 0.110 & 0.051 & -0.109 & 0.101 & 0.020 & 0.101 & 325.4 \\
F & 0.051 & -0.110 & 0.122 & 0.061 & -0.121 & 0.069 & 0.020 & 0.101 & 505.2 \\
\hline
\end{tabular}

\section{References}

Akaike, H. (1974). A new look at the statistical model identification. IEEE Transactions on Automatic Control, AC-19, 716-723. doi:10.1109/TAC.1974.1100705

Ashby, F. G. A. (1983). A biased random-walk model for two choice reaction times. Journal of Mathematical Psychology, 27, 277-297.

Ashby, F. G., \& Townsend, J. T. (1980). Decomposing the reaction time distribution: Pure insertion and selective influence revisited. Journal of Mathematical Psychology, 21, 93-123. doi:10.1016/ 0022-2496(80)90001-2

Busemeyer, J. R., \& Diederich, A. (2002). Survey of decision field theory. Mathematical Social Sciences, 43, 345-370.

Chiew, K. S., \& Braver, T. S. (2011). Monetary incentives improve performance, sometimes: Speed and accuracy matter, and so might preparation. Frontiers in Psychology, 2, 325. doi:10.3389/fpsyg.2011.00325

Cousineau, D., Brown, S., \& Heathcote, A. (2004). Fitting distributions using maximum likelihood: Methods and packages. Behavior Research Methods, Instruments, \& Computers, 36, 742-756. doi:10.3758/BF03206555

Dambacher, M., Hübner, R., \& Schlösser, J. (2011). Monetary incentives in speeded perceptual decision: Effects of penalizing errors versus slow responses. Frontiers in Psychology, 2, 248. doi:10.3389/fpsyg.2011.00248

Diederich, A. (1995). Intersensory facilitation of reaction time: Evaluation of counter and diffusion coactivation models. Journal of Mathematical Psychology, 39, 197-215.

Diederich, A. (1997). Dynamic stochastic models for decision making under time constraints. Journal of Mathematical Psychology, 41, 260-274.

Diederich, A. (2008). A further test of sequential-sampling models that account for payoff effects on response bias in perceptual decision tasks. Perception \& Psychophysics, 70, 229-256.

Diederich, A., \& Busemeyer, J. R. (2006). Modeling the effects of payoff on response bias in a perceptual discrimination task: Bound-change, drift-rate-change, or two-state-processing hypothesis. Perception \& Psychophysics, 68, 194-207. doi:10.3758/ BF03193669
Edwards, W. (1965). Optimal strategies for seeking information: Models for statistics, choice reaction times, and human information processing. Journal of Mathematical Psychology, 2, 312-329. doi:10.1016/0022-2496(65)90007-6

Falmagne, J.-C. (1965). Stochastic models for choice reaction time with applications to experimental results. Journal of Mathematical Psychology, 2, 77-124.

Fitts, P. M. (1966). Cognitive aspects of information processing: III. Set for speed versus accuracy. Journal of Experimental Psychology, 71, 849-857. doi:10.1037/h0023232

Green, D. M., \& Swets, J. A. (1966). Signal detection and psychophysics. New York, NY: Wiley.

Heath, R. A. (1981). A tandem random walk model for psychological discrimination. British Journal of Mathematical and Statistical Psychology, 34, 76-92.

Heath, R. A. (1992). A general nonstationary diffusion model for twochoice decision making. Mathematical Social Sciences, 23, 283309.

Heathcote, A., Brown, S., \& Cousineau, D. (2004). QMPE: Estimating Lognormal, Wald, and Weibull RT distributions with a parameterdependent lower bound. Behavior Research Methods, Instruments, \& Computers, 36, 277-290. doi:10.3758/BF03195574

Jarvik, M. E. (1951). Probability learning and a negative recency effect in the serial anticipation of alternative symbols. Journal of Experimental Psychology, 41, 291-297.

Kirby, N. H. (1976). Sequential effects in two-choice reaction time: Automatic facilitation or subject expectancy? Journal of Experimental Psychology. Human Perception and Performance, 2, 567-577.

LaBerge, D. (1962). A recruitment theory of simple behavior. Psychometrika, 27, 375-396.

Lagarias, J. C., Reeds, J. A., Wright, M. H., \& Wright, P. E. (1998). Convergence properties of the Nelder-Mead simplex method in low dimensions. SIAM Journal on Optimization, 9, 112-147.

Laming, D. R. J. (1968). Information theory of choice-reaction times. New York, NY: Academic Press.

Laming, D. R. J. (1969). Subjective probability in choice-reaction experiments. Journal of Mathematical Psychology, 6, 81-120. 
Laming, D. (2010). Statistical information and uncertainty: A critique of applications in experimental psychology. Entropy, 12, 720 771. doi: $10.3390 / \mathrm{e} 12040720$

Leite, F. P., \& Ratcliff, R. (2010). Modeling reaction time and accuracy of multiple-alternative decisions. Attention, Perception, \& Psychophysics, 72, 246-273. doi:10.3758/APP.72.1.246

Leite, F. P., \& Ratcliff, R. (2011). What cognitive processes drive response biases? A diffusion model analysis. Judgment and Decision Making, 6, 651-687. Retrieved from http://journal.sjdm.org/vol6.7.html

Link, S. W., \& Heath, R. A. (1975). A sequential theory of psychological discrimination. Psychometrika, 40, 77-105.

Luce, R. D. (1986). Response times: Their role in inferring elementary mental organization. New York, NY: Oxford University Press.

Maddox, W. T., \& Bohil, C. J. (1998). Base-rate and payoff effects in multidimensional perceptual categorization. Journal of Experimental Psychology: Learning, Memory, and Cognition, 24, 1459-1482. doi:10.1037/0278-7393.24.6.1459

Mulder, M. J., Wagenmakers, E.-J., Ratcliff, R., Boekel, W., \& Forstmann, B. U. (2012). Bias in the brain: A diffusion model analysis of prior probability and potential payoff. Journal of Neuroscience, 32, 23352343.

Nelder, J. A., \& Mead, R. (1965). A simplex method for function minimization. The Computer Journal, 7, 308-313. doi:10.1093/ comjn1/7.4.308

Posner, M. I. (1978). Chronometric explorations of mind. Hillsdale, NJ: Erlbaum.

Ratcliff, R. (1978). A theory of memory retrieval. Psychological Review, 85, 59-108. doi:10.1037/0033-295X.85.2.59

Ratcliff, R. (1980). A note on modeling accumulation of information when the rate of accumulation changes over time. Journal of Mathematical Psychology, 21, 178-184.
Ratcliff, R. (1981). A theory of order relation in perceptual matching. Psychological Review, 88, 552-572.

Ratcliff, R., \& Murdock, B. B., Jr. (1976). Retrieval processes in recognition memory. Psychological Review, 83, 190-214. doi:10.1037/0033-295X.83.3.190

Ratcliff, R., \& Rouder, J. F. (1998). Modeling response times for twochoice decisions. Psychological Science, 9, 347-356. doi:10.1111/1467-9280.00067

Ratcliff, R., \& Smith, P. L. (2004). A comparison of sequential sampling models for two-choice reaction time. Psychological Review, 111, 333-367.

Ratcliff, R., Thapar, A., \& McKoon, G. (2001). The effects of aging on reaction time in a signal detection task. Psychology and Aging, 16, 323-341. doi:10.1037/0882-7974.16.2.323

Remington, R. J. (1969). Analysis of sequential effects in choice reaction times. Journal of Experimental Psychology, 82, 250-257.

Simen, P., Contreras, D., Buck, C., Hu, P., Holmes, P., \& Cohen, J. D. (2009). Reward rate optimization in two-alternative decision making: Empirical tests of theoretical predictions. Journal of Experimental Psychology: Human Perception and Performance, 35, 1865-1897. doi:10.1037/a0016926

Stone, M. (1960). Models for choice reaction time. Psychometrika, 25, 251-260.

Swets, J. A., Tanner, W. P., Jr., \& Birdsall, T. G. (1961). Decision processes in perception. Psychological Review, 68, 301-340. doi: $10.1037 / \mathrm{h} 0040547$

Van Zandt, T., \& Ratcliff, R. (1995). Statistical mimicking of reaction time data: Single-process models, parameter variability, and mixtures. Psychonomic Bulletin \& Review, 2, 20-54. doi:10.3758/ BF03214411

Vickers, D. (1970). Evidence for an accumulator of psychophysical discrimination. Ergonomics, 13, 37-58. 